\title{
Autophagy facilitates the development of resistance to the tumor necrosis factor superfamily member TRAIL in breast cancer
}

\author{
SHANGGE $\mathrm{LV}^{1,2}$, XIAOLONG WANG ${ }^{1}$, NING ZHANG ${ }^{1}$, MINGJUAN SUN ${ }^{1}$, \\ WENWEN QI ${ }^{2}$, YAMING $\mathrm{LI}^{2}$ and QIFENG YANG ${ }^{1,3}$ \\ ${ }^{1}$ Department of Breast Surgery, Qilu Hospital, Shandong University, Jinan, Shandong 250012; \\ ${ }^{2}$ School of Medicine, Shandong University, Jinan, Shandong 250012; ${ }^{3}$ Department of Pathology Tissue Bank, \\ Qilu Hospital, Shandong University, Jinan, Shandong 250012, P.R. China
}

Received October 21, 2014; Accepted December 12, 2014

DOI: $10.3892 /$ ijo.2014.2812

\begin{abstract}
Autophagy, an important homeostatic cellular recycling mechanism, has emerged as a novel cytoprotective mechanism to increase tumor cell survival through escaping chemotherapy-induced cell death. To explore whether autophagy plays a protective role in the resistance to the tumor necrosis factor-related apoptosis-inducing ligand (TRAIL), we evaluated the autophagy levels in TRAIL-sensitive MDA-MB-231 breast cancer cell lines and in TRAIL-refractory MDA-MB-231 cells before and after TRAIL treatment. After treatment with 40 ng/ml TRAIL, TRAIL-sensitized MDA-MB-231 parental cells expressed higher level of LC3B protein and accumulated more autophagic vacuoles. Compared with TRAIL-sensitive MDA-MB-231, MDA-MB-231 TRAIL-refractory cells showed higher levels of the lipidated form of LC3B and decreased p62/SQSTM1 protein expression, characterizing the occurrence of increased autophagic flux in TRAIL-refractory cells. Electron microscopy and monodansylcadaverine (MDC) autophagy-specific fluorescence staining analyses also revealed that the accumulation of autophagic vacuoles was drastically higher in TRAIL-refractory MDA-MB-231 parental cells. We demonstrated that chloroquine (CQ) and 2-(4-morpholinyl)-8-phenylchromone (LY294002) could effectively reduce TRAIL-refractory breast cancer cell viability. Combination of TRAIL with CQ could effectively reverse the resistance of MDA-MB-231 TRAIL-refractory cells to TRAIL. Knockdown of light chain 3 (LC3) expression via small interfering RNA (siRNA) similarly resulted in reduced TRAIL-refractory cell proliferation and re-sensitizing to TRAIL. This is the first report showing that breast cancer cells chronically exposed to TRAIL exhibit upregulation of
\end{abstract}

Correspondence to: Professor Qifeng Yang, Department of Breast Surgery, Qilu Hospital, Shandong University, Wenhua Xi Road No. 107, Jinan, Shandong 250012, P.R. China

E-mail: qifengy@gmail.com

Key words: TRAIL, drug resistance, reverse, autophagy, breast cancer the autophagic activity, indicating that autophagy efficiently protects breast cancer cells from TRAIL. Therapeutic targeting of autophagosome formation could be a novel molecular avenue to reduce the resistance of TRAIL in breast cancer.

\section{Introduction}

Autophagy is an evolutionarily conserved catabolic process. It can damage long-lived cellular proteins and degrade organelles, through facilitating cytoplasmic turnover and maintains metabolic homeostasis in double-membrane vesicles, termed autophagosomes $(1,2)$. When cells need to generate intracellular nutrients and energy, for instance, during starvation, growth factor withdrawal, or high bioenergetic demands, autophagy can be upregulated (3). Moreover, basal autophagy can serve as an important homeostatic cellular recycling mechanism responsible for degrading unnecessary or dysfunctional cellular organelles and proteins in all living cells (4). Autophagy can promote the survival of tumor cells in poorly vascularized and hypoxic tumors or cytotoxic treatments $(5,6)$. Many preclinical studies have demonstrated that genetic or pharmacological inhibition of autophagy can enhance drug- and radiation-induced cytotoxicity in cell culture and in vivo (7). Autophagy has a potent cytoprotective survival pathway in normal and cancer cells.

Tumor necrosis factor-related apoptosis-inducing ligand (TRAIL) belongs to the TNF super family that can initiate apoptosis via activating the extrinsic apoptosis pathway (8). Due to its remarkable feature of selectively inducing apoptosis in cancer cells without causing damage to normal cells (9), has led to multiple clinical trials to evaluate the antitumor potential of recombinant human TRAIL (rhTRAIL) and it emerged as a potential therapeutic agent $(10,11)$. TRAIL triggers typical apoptotic signaling by binding to its receptors, death receptor 4 (DR4) and 5 (DR5), thereby recruiting the assembly of the death-inducing signaling complex (DISC), which activates the caspase cascade (12). Ongoing and completed phase I and II clinical trials with TRAIL are showing clinically promising outcomes with no apparent toxicity (13). However, recent studies have indicated that a 
variety of cancer cells are resistant to the apoptotic effects of TRAIL $(14,15)$. The majority of breast cancer cells are resistant to TRAIL-mediated apoptosis (16). The mechanisms underlying resistance to TRAIL are not fully-understood, and the identification of TRAIL resistance factors could facilitate the development of more effective TRAIL-based cancer therapies.

We demonstrated that autophagy may be a potential target to overcome TRAIL resistance of breast cancer cells.

\section{Materials and methods}

Production of rhTRAIL. rhTRAIL was produced by our laboratory $(17,18)$.

Cell culture and reagents. The human breast cancer cell line MDA-MB-231 was obtained from the American Type Culture Collection (ATCC) (Manassas, VA, USA), and routinely cultured in DMEM medium (Gibco-BRL, Rockville, MD, USA)/high glucose medium supplemented with $10 \%$ FBS (Haoyang Biological Manufacturer Co., Ltd., Tianjin, China) containing, $100 \mathrm{U} / \mathrm{ml}$ penicillin and $100 \mu \mathrm{g} / \mathrm{ml}$ streptomycin. The cells were maintained at $37^{\circ} \mathrm{C}$ in humidified air with $5 \% \mathrm{CO}_{2}$. Chloroquine (CQ) and monodansylcadaverine (MDC) were obtained from Sigma-Aldrich (St. Louis, MO, USA).

Establishment of TRAIL-acquired autoresistance in MDA-MB-231 breast cancer cells. To establish MDA-MB-231 TRAIL-refractory cells exhibiting resistance to the TRAIL, TRAIL-sensitive MDA-MB-231 parental cells were exposed to incremental increases of TRAIL. TRAIL-resistance selection continued until the MDA-MB-231 cells could sustain cell viability and proliferate when challenged with $400 \mathrm{ng} / \mathrm{ml}$. TRAIL-refractory cells were obtained upon exposure of MDA-MB-231 cells for a minimum of 10 months before starting any experimental procedure. Briefly, MDA-MB-231 cells that were initially exposed to $40 \mathrm{ng} / \mathrm{ml}$ TRAIL for 3 months, and then treated with $400 \mathrm{ng} / \mathrm{ml}$ TRAIL for 2 months (twice weekly) resisted continuous growth in $640 \mathrm{ng} / \mathrm{ml} \mathrm{TRAIL}$. The resistant cells were maintained in medium without TRAIL for at least 10 days before each experiment.

Cell viability assay. Cell viability was determined by colorimetric assay using 3-(4,5-dimethylthiazol-2-yl)-2,5-diphenyltetrazolium bromide (MTT). Briefly, cells (2,000 cells/well) were seeded in 96-well plates and allowed to attach overnight at $37^{\circ} \mathrm{C}$. Then culture medium containing vehicle or drugs was added to the medium in each well and incubating for indicated time points. The cells in 96 -well plates were incubated with $20 \mu \mathrm{l}$ MTT in growth medium at indicated time points. After incubating at $37^{\circ} \mathrm{C}$ for $4 \mathrm{~h}$, the supernatants were carefully aspirated and the resulting crystals were dissolved in $100 \mathrm{ml}$ dimethyl sulfoxide (DMSO). Absorbance values at $490 \mathrm{~nm}$ were determined by the Microplate Reader (Bio-Rad, Hercules, CA, USA). Data are presented as the percentage of survival rate relative to vehicle-treated control.

Electron microscopy assay. To detect the autophagic vacuoles directly, we performed ultra structural analysis under electron microscopy. Briefly, cells were fixed in a mixture of $2.5 \%$, paraformaldehyde and $2.0 \%$ gluteraldehyde in $0.1 \mathrm{M}$ cacodylate buffer, $\mathrm{pH} 7.3$, for $1 \mathrm{~h}$. After fixation, the samples were post-fixed in $1 \% \mathrm{OsO}_{4}$ in the same buffer for $1 \mathrm{~h}$ and then subjected to electron microscopic analysis. Representative areas were selected for ultrathin sectioning and viewed with a JEM 1010 transmission electron microscope (JEOL USA, Inc., Peabody, MA, USA) at an accelerating voltage of $80 \mathrm{kV}$. Digital images were obtained with the AMT Imaging System (Advanced Microscopy Techniques, Danvers, MA, USA).

Immunofluorescence staining. Immunofluorescence staining was used to perform the localization and the level of expression of LC3B and p62/SQSTM1. In brief, cells were grown on cover slips in the 24-well plates for 3 days, for the different treatments. After fixing with $4 \%$ paraformaldehyde for $15 \mathrm{~min}$ at room temperature and extensive wash in PBS, cells were permeabilized with $0.1 \%$ Triton X-100 in PBS (PBST) for $25 \mathrm{~min}$. Then cells were blocked with $10 \%$ goat serum in PBST for $1 \mathrm{~h}$, followed by incubating with rabbit anti-LC3B or anti-p62/SQSTM1 monoclonal antibodies (Cell Signaling Technology, Inc., Danvers, MA, USA) and rhodamine-conjugated anti-rabbit secondary antibodies (Kirkegaard \& Perry Laboratories, Inc., Gaithersburg, MD, USA). The nuclear DNA was stained with 4',6-Diamidino-2-phenylindole (DAPI) for $5 \mathrm{~min}$. Finally, antifading medium was added and the cover slips were immediately observed under a DP71 fluorescence microscope (Olympus, Tokyo, Japan).

MDC staining. MDC staining of autophagic vacuoles was performed for autophagy analysis. Autophagic vacuoles were labeled with $\mathrm{MDC}(50 \mu \mathrm{M})$ in $\mathrm{PBS}$ at $37^{\circ} \mathrm{C}$ for $10 \mathrm{~min}$. And then, the cells were washed three times with PBS. Autophagic vacuoles were observed immediately under DP71 fluorescence microscope (excitation wavelength, $380 \mathrm{~nm}$; emission filter, $525 \mathrm{~nm})$.

Immunoblotting analysis. Vehicle- or drug-treated cells were lysed in a lysis buffer containing $50 \mathrm{mM}$ Tris- $\mathrm{HCl}, \mathrm{pH} 7.5$, $150 \mathrm{mM} \mathrm{NaCl}, 1 \%$ Nonidet P-40, 0.25\% sodium deoxycholate, $0.1 \%$ SDS with protease inhibitors. Lysates were centrifuged at $12,000 \mathrm{rpm}$ for $15 \mathrm{~min}$. Supernatants were collected, subjected to electrophoresis on $12 \%$ (for LC3B antibody) or $10 \%$ (for other primary antibody) SDS-polyacrylamide gels and transferred to polyvinylidene fluoride membranes (ImmobilonP; Millipore, Bedford, MA, USA). The membrane was blocked with $5 \%$ non-fat dry milk for $1 \mathrm{~h}$, then incubated with indicated primary antibody (Cell Signaling Technology, Inc.) overnight at $4^{\circ} \mathrm{C}$. The membrane was treated with horseradish peroxidase conjugated secondary antibodies. Signals were detected by enhanced chemiluminescence.

siRNA transfection. The synthetic small interfering RNA (siRNA) oligos-specific to light chain 3 (LC3) was purchased from Shanghai GenePharma, Ltd. (Shanghai, China) with the corresponding sequence: 5'-AAAUCCCGG UGAUAAUAGA-3'. Cells were seeded in 6-well plates at a density of $6 \times 10^{5}$ cells/well in antibiotic-free medium and allowed to attach overnight. siRNA and transfection reagent were diluted, respectively, in a separate tube containing $200 \mu \mathrm{l}$ 
of serum-free medium. Following 5 min incubation, siRNA-containing medium was added to DharmaFect-containing medium. This mixture was allowed to incubate for 20 min to allow liposome formation and siRNA loading. Antibiotic-free complete medium was then added to a final volume of $2 \mathrm{ml}$ and plated onto cells. Transfected cells were split 1:2 $48 \mathrm{~h}$ later into new 6-well plates and allowed to attach overnight. The next day, cells were retransfected exactly as before, so as to achieve more efficient and sustained knockdown for an extended period of time. Cells were re-seeded $24 \mathrm{~h}$ later for subsequent experimentation.

Statistical analysis. SPSS software version 18.0 was used for statistical analysis. Variance analysis was used to determine significance. All error bars represent the SD of three experiments. Differences with $\mathrm{p}<0.05$ were considered significant.

\section{Results}

TRAIL reduced cell viability and induced autophagy in breast cancer. To assess the effects of TRAIL on breast cancer cell viability and to examine whether TRAIL-refractory cells were resistant to TRAIL, MDA-MB-231 cells and MDA-MB-231 TRAIL-refractory cell lines were treated with varying doses of TRAIL $(0,10,20,40,80,160 \mathrm{ng} / \mathrm{ml}$ and $0,20,40,80,160$, $320,640,1280 \mathrm{ng} / \mathrm{ml}$, respectively) for $72 \mathrm{~h}$. Drug-induced cell death was measured on MTT assay. TRAIL resistance was demonstrated in the TRAIL-refractory cells (Fig. 1A).

The TRAIL-refractory cells were separately examined for the morphological changes of MDA-MB-231 cells and MDA-MB-231 TRAIL-refractory cells by light microscopy. We observed that the cells were larger after treatment with TRAIL (Fig. 1B).

To find the mechanisms involved in TRAIL-mediated cell death, we investigated the autophagic pathway.

The MDA-MB-231 and MDA-MB-231 TRAIL-refractory cells were treated with TRAIL (40 or $400 \mathrm{ng} / \mathrm{ml}$ ) for $72 \mathrm{~h}$. The percentages of autophagic vacuoles in tested cell lines following treatment with TRAIL were analyzed by LC3B conversion by immunoblotting and immunofluorescence staining. As shown in Fig. 2A, LC3B immunoblotting revealed that LC3B-I (16 kDa) and LC3-II (14 kDa), LC3B-lipidated form was increased in a dose-dependent manner in MDA-MB-231 and MDA-MB-231 TRAIL-refractory cells following TRAIL treatment. High levels of LC3-lipidated form indicated impairment in autophagosome maturation. Fluorescence micrographs (Fig. 2B) revealed the number and intensity of punctuate LC3B fluorescence increased after treatment with TRAIL, we utilized this property of LC3B to initially monitor changes in the dynamics of the autophagic process. The results showed, LC3B-lipidated form increased in a dose-dependent manner in breast cancer cells following TRAIL treatment.

To detect the development of autophagic vacuoles, non-treated and treated MDA-MB-231 or MDA-MB-231 TRAIL-refractory cells were stained with MDC. MDC is a specific marker for autophagic vacuoles (19). The result showed TRAIL-induced autophagic vacuoles formation in breast cancer cells when compared with control cells (Fig. 2C).
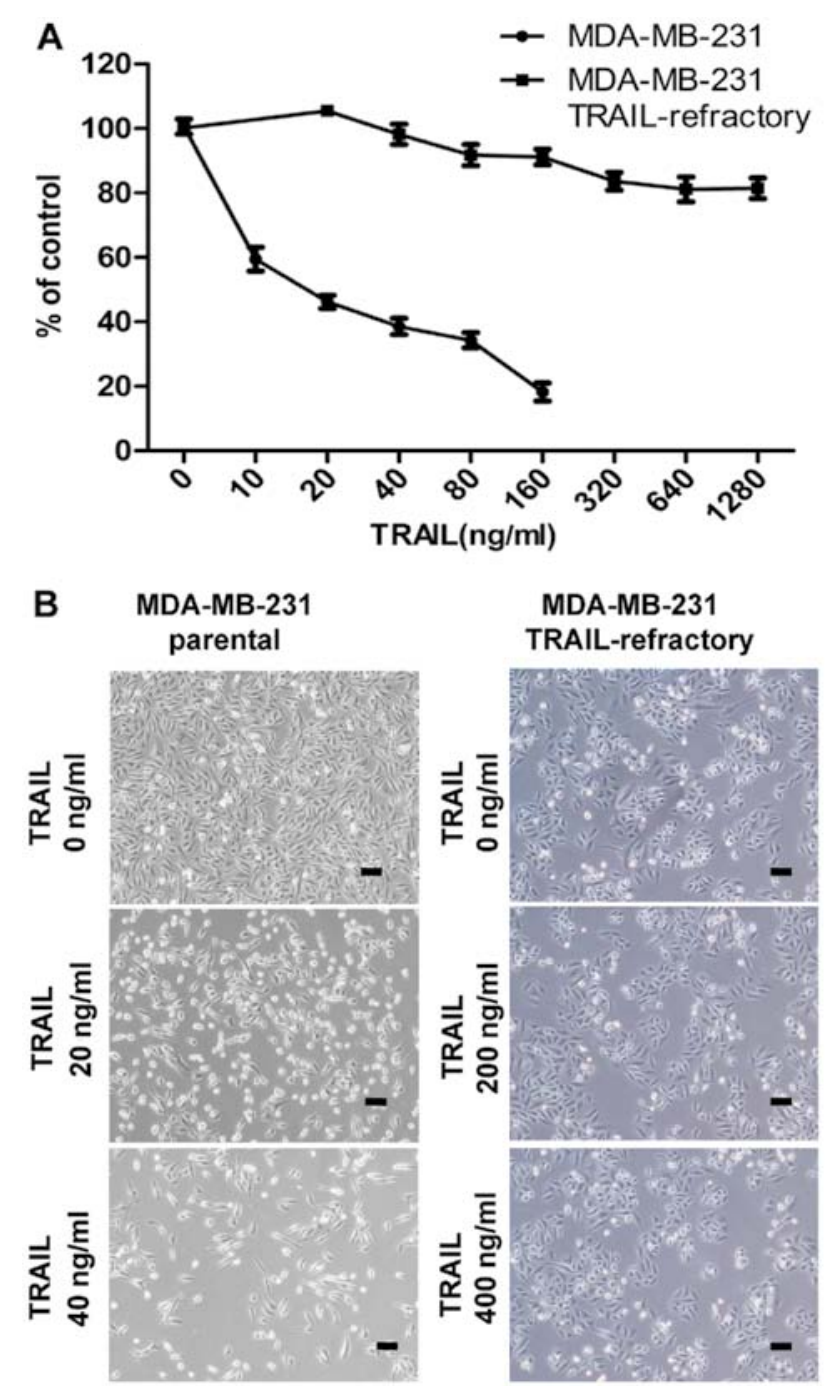

Figure 1. Tumor necrosis factor-related apoptosis-inducing ligand (TRAIL) reduced cell viability and triggered intracellular vesicular organelles in breast cancer cells. (A) The cytotoxic effect of TRAIL was measured by 3-(4,5-dimethylthiazol-2-yl)-2,5-diphenyltetrazolium bromide (MTT) assay. MDA-MB-231 parental cells and TRAIL-refractory cells were treated with different concentrations of TRAIL for $72 \mathrm{~h}$ and subjected to MTT assay. The viability of untreated cells was $100 \%$. The experiments were performed in triplicate and data presented as the mean $\pm \mathrm{SD}$ of three separate experiments. (B) Representative microphotographs of untreated and experimental cell cultures following $24 \mathrm{~h}$ exposure to TRAIL concentrations as high as 20,40 or $200,400 \mathrm{ng} / \mathrm{ml}$. Scale bar, $20 \mu \mathrm{m}$.

TRAIL resistance correlated with an accumulation of autophagosomes. Autophagic activity might represent a previously unrecognized pro-survival pathway underlying acquired autoresistance to TRAIL. Since it is a dynamic, multi-step process that can be modulated at several steps, both positively and negatively, we examined autophagy in several ways. Firstly, the striking accumulation of autophagosomes was measured by LC3 lipidation on western blotting and fluorescent staining. As shown in Fig. 3A, immunoblotting exhibited distinct patterns of LC3B expression between TRAIL-sensitive and TRAIL-refractory cell lines. In TRAIL-sensitive cell lines, LC3B existed primarily in its cytosolic form, LC3B-I. By contrast, TRAIL-refractory cells were characterized by the upregulating of the lipidated form, LC3B-II. Using fluorescence microscopy, we confirmed the high basal levels of autopha- 
A

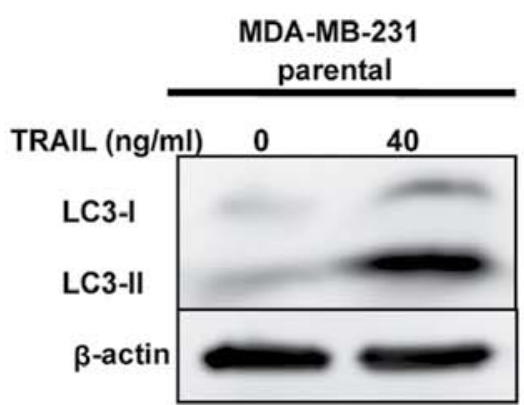

B
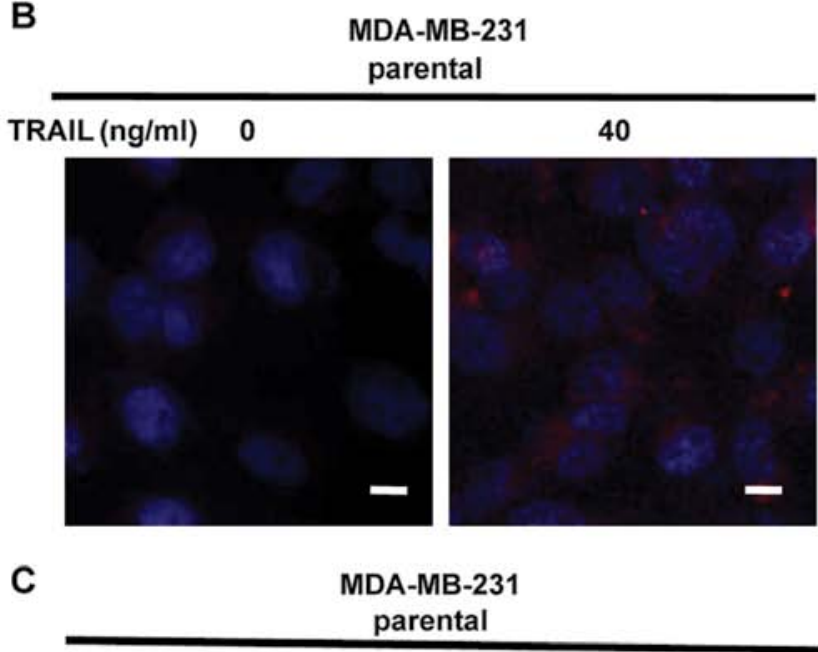

$\operatorname{TRAIL}(\mathrm{ng} / \mathrm{ml}) \quad 0$

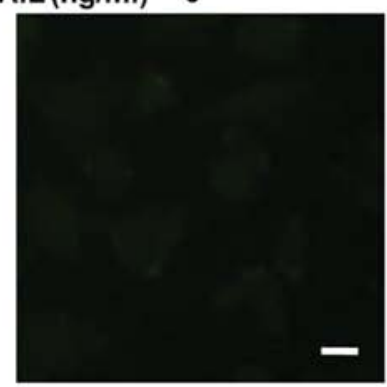

40

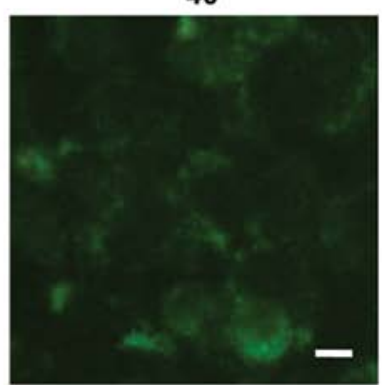

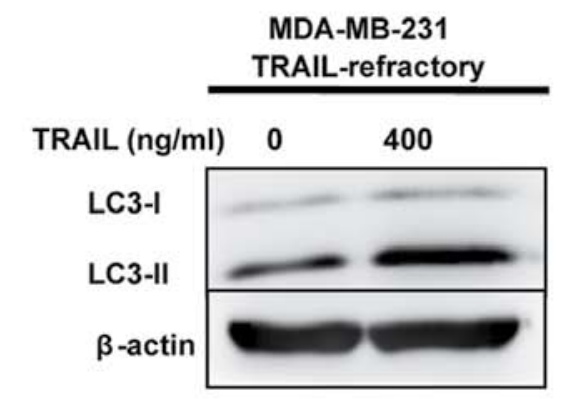

MDA-MB-231

TRAIL-refractory

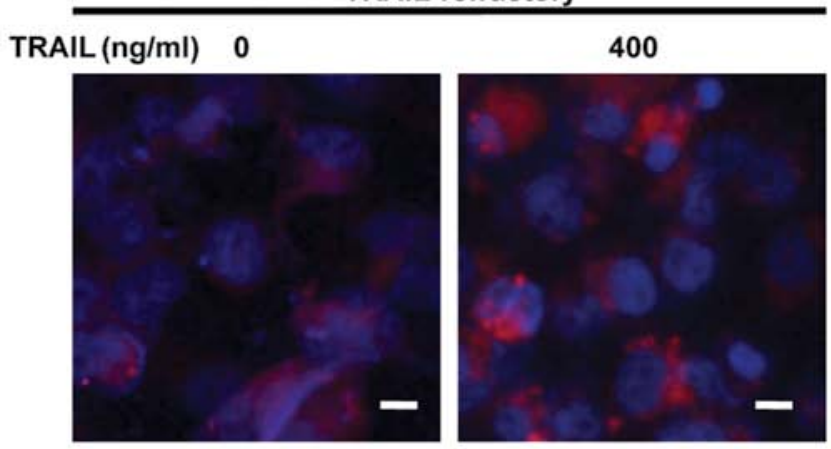

MDA-MB-231

TRAIL-refractory

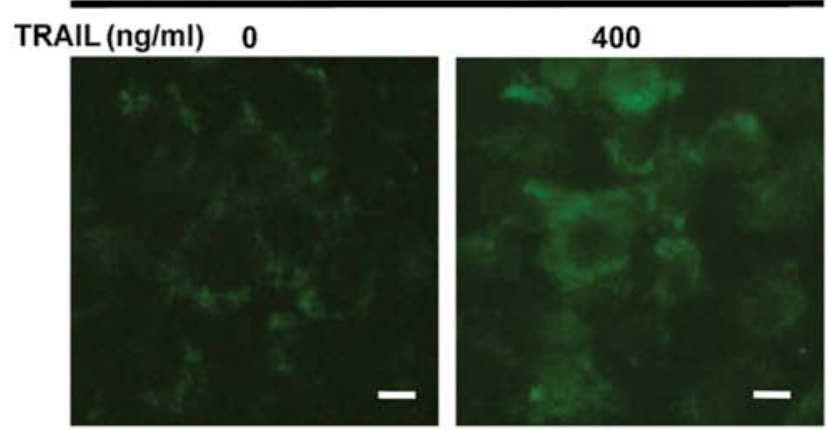

Figure 2. Tumor necrosis factor-related apoptosis-inducing ligand (TRAIL) induces autophagy in MDA-MB-231 parental and MDA-MB-231 TRAIL-refractory cells. (A) MDA-MB-231 parental cell lysates were harvested with $40 \mathrm{ng} / \mathrm{ml}$ of TRAIL for $72 \mathrm{~h}$. MDA-MB-231 TRAIL-refractory cells were harvested with $400 \mathrm{ng} / \mathrm{ml}$ of TRAIL for $72 \mathrm{~h}$. $\beta$-actin was used as a loading control. (B) Aggregation of LC3B in TRAIL-treated cells. Cells were treated with or without 40 or $400 \mathrm{ng} / \mathrm{ml}$ TRAIL, and then stained with the LC3B antibodies using immunofluorescence staining. Puncta represent the autophagosome formation. Scale bar, $10 \mu \mathrm{m}$. (C) Acidic vesicular organelles induced by TRAIL were stained with monodansylcadaverine (MDC). Scale bar, $10 \mu \mathrm{m}$. Results shown are representative of three independent experiments.

gosomes in TRAIL-resistant cell lines. As shown in Fig. 3B, TRAIL-refractory cells exhibited a significant increase of LC-3B per individual cell when compared with MDA-MB-231 cells, indicating that MDA-MB-231 TRAIL-refractory cells exhibited punctuate structures that are typical features of autophagosomes.

Moreover, we performed two complementary experimental strategies by measuring p62/SQSTM1 protein expression to further distinguish the level of autophagy. As shown in Fig. 3A and C, immunoblotting and immunofluorescence staining detected a slight but significant reduction in the total p62/SQSTM1 protein content in TRAIL-refractory-derived whole cell lysates when compared with $\mathrm{p} 62 / \mathrm{SQSTM} 1$ protein expression status in TRAIL-naïve MDA-MB-231 parental cells.
We used MDC staining and electron microscopy to further confirm that autophagosome formation was increased in TRAIL-refractory cells. We demonstrated that TRAIL-refractory cells exhibited higher fluorescent density and more MDC-labeled particles compared with TRAIL-naïve MDA-MB-231 cells, indicating that TRAIL resistance correlates with the increase of MDC recruitment to autophagosomes in the cytoplasm of cells (Fig. 3D). Further, electron microscopy images clearly showed the presence of a large number of autophagosomes in TRAIL-refractory cells but not in MDA-MB-231 cells (Fig. 3E). These results suggested that TRAIL resistance was related to an accumulation of autophagosomes.

Blocking autophagosome function enhances TRAIL efficacy in TRAIL-refractory cells. To pharmacologically evaluate 
A

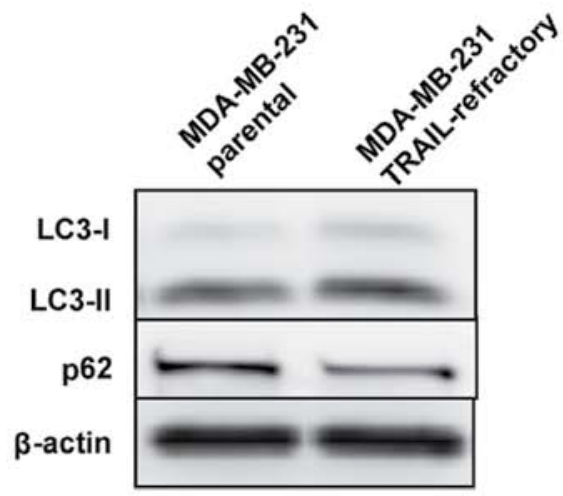

B

MDA-MB-231 parental

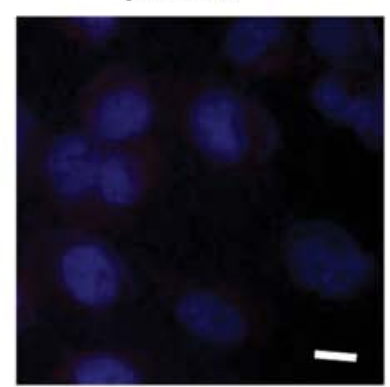

D
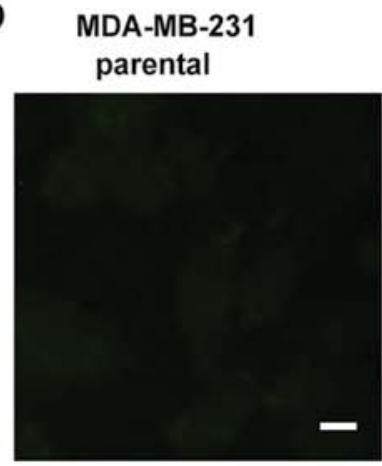

MDA-MB-231 TRAIL-refractory

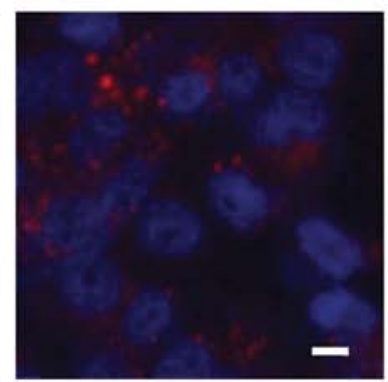

MDA-MB-231 TRAIL-refractory

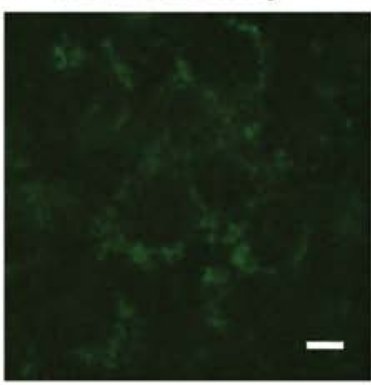

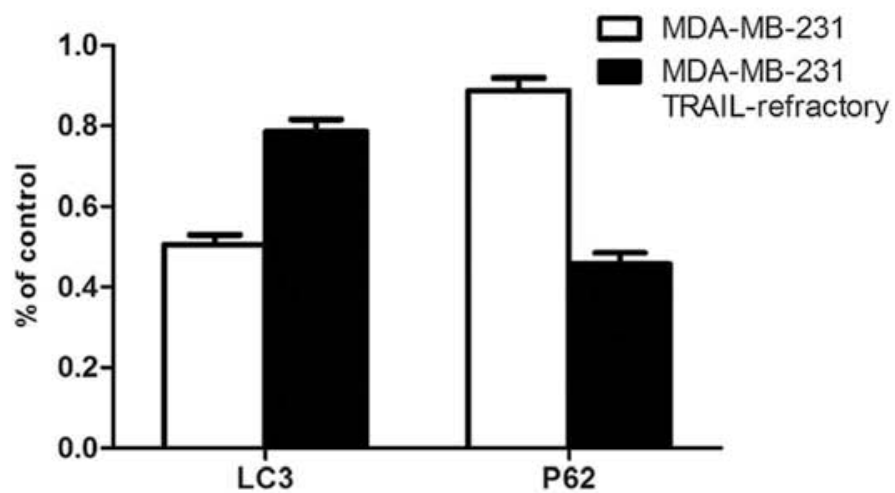

C

MDA-MB-231 parental

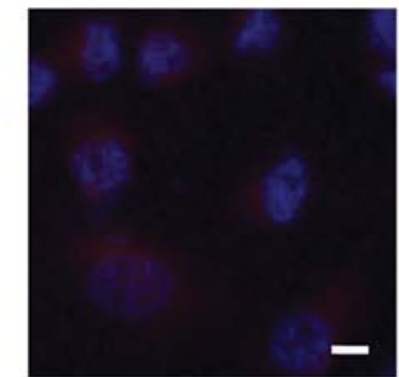

E

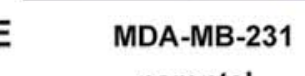

parental

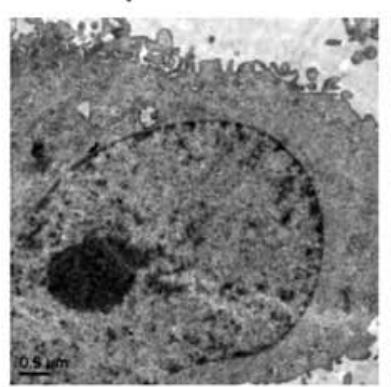

MDA-MB-231

TRAIL-refractory

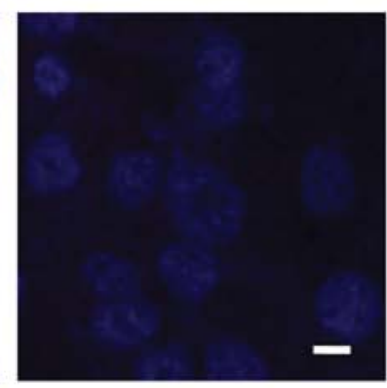

MDA-MB-231 TRAIL-refractory

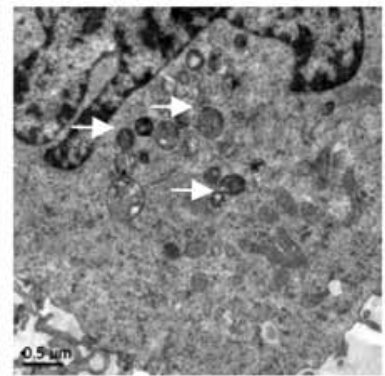

Figure 3. Dynamics of autophagosome formation in MDA-MB-231 parental cells and MDA-MB-231 tumor necrosis factor-related apoptosis-inducing ligand (TRAIL)-refractory cells. Autophagosome formation in whole cell lysates of TRAIL-naïve MDA-MB-231 parental cells and TRAIL-refractory used LC3B and p62 antibodies. (A) Cells were harvested and analyzed by western blotting. $\beta$-actin was detected as a loading control. (B) After fixation and permeabilization, cellular distribution of autophagosome marker light chain 3 (LC3) was assessed by immunofluorescence. LC3 antibody and 4',6-diamidino-2-phenylindole (DAPI) for nuclear counter staining. Scale bar, $10 \mu \mathrm{m}$. (C) After fixation and permeabilization, cellular distribution of p62 was assessed following staining with a p62 antibody and DAPI for nuclear counterstaining. Scale bar, $10 \mu \mathrm{m}$. (D) Monodansylcadaverine (MDC) staining was used to analyse the acidic vesicular organelles in MDA-MB-231 and MDA-MB-231 TRAIL-refractory cells. Scale bar, $10 \mu \mathrm{m}$. (E) Representative electron micrographs of MDA-MB-231 and MDA-MB-231 TRAIL-refractory cells. Arrows indicate autophagic vacuoles. Scale bar, $0.5 \mu$ m. Equivalent results were obtained in three independent experiments.

whether the basal autophagy was actively involved in the development of TRAIL resistance, we assessed the growth inhibitory effects of autophagy inhibitors (20). Firstly, TRAIL-resistant MDA-MB-231, TRAIL-refractory and TRAIL-naïve MDA-MB-231 cells were pretreated with CQ. The cytotoxic effect of CQ treatment was measured by MTT. The result showed that CQ effectively reduced cell viability in TRAIL-refractory cells (Fig. 4A). This was further supported when similar studies were carried out in the presence of 2-(4-morpholinyl)-8-phenylchromone (LY294002). In terms of cell viability, TRAIL-refractory cells were exquisitely sensitive to this agent that blocks phosphatidy-linositol 3-kinase activity and prevents autophagic sequestration (Fig. 4A).
Pharmacologically-induced loss of autophagosome formation is highly cytotoxic to TRAIL-refractory cells (Fig. 4A) compared with cell viability effects in TRAIL-naïve MDA-MB-231 parental cells (Fig. 4B). Representative immune-confocal images of MDA-MB-231 TRAIL-refractory cells cultured in the absence or presence of CQ $(20 \mathrm{ng} / \mathrm{ml})$ and LY294002 $(10 \mu \mathrm{M})$ for $72 \mathrm{~h}$ are shown in Fig. 4D. We further confirmed that LY294002 treatment was efficient at reducing the number of LC3-positive autophagosomes while CQ was able to increase the number of LC3-positive autophagosomes in TRAIL-refractory cells. Collectively, these findings strongly suggested that increased macroautophagy actively provided a survival function to TRAIL-refractory cells. 

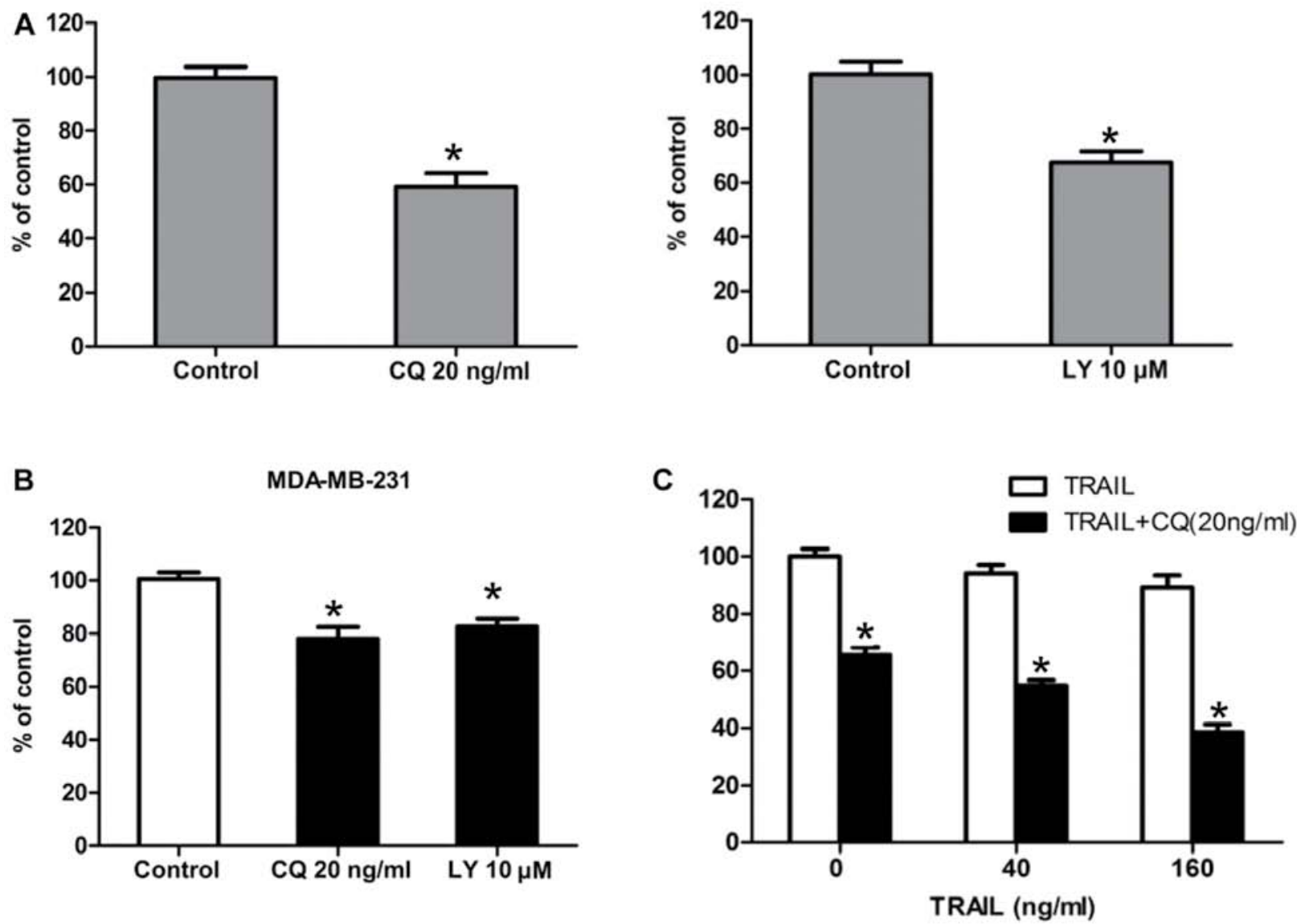

D

MDA-MB-231 TRAIL-refractory

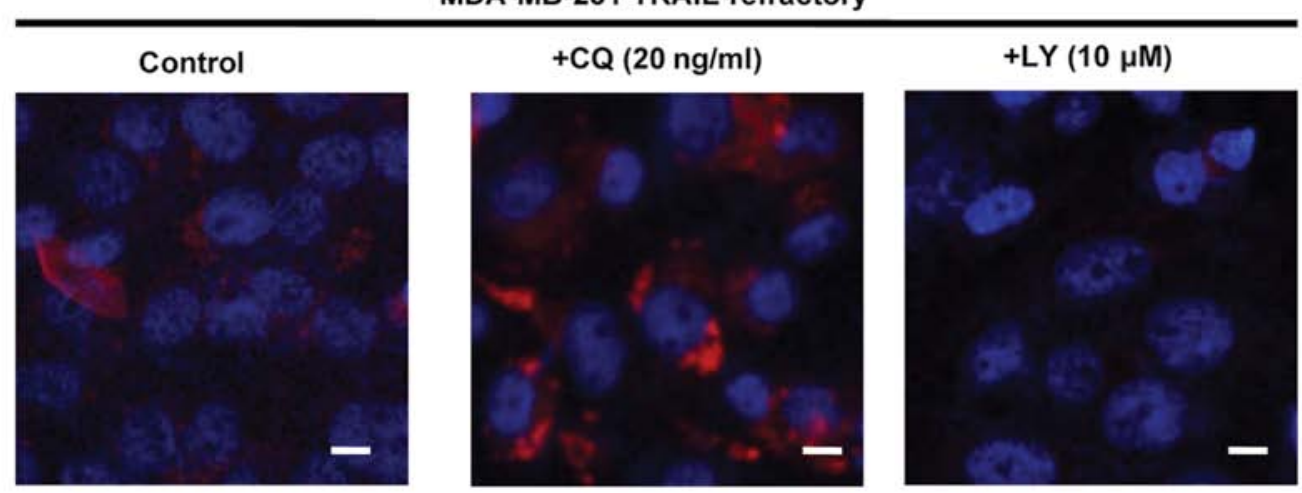

Figure 4. Changes in the cell viability of tumor necrosis factor-related apoptosis-inducing ligand (TRAIL)-refractory cells upon pharmacological modulation of autophagosomes formation. (A and B) The metabolic status of MDA-MB-231 parental cells and TRAIL-refractory cells treated with autophagy inhibitors including chloroquine (CQ), LY294002 for $72 \mathrm{~h}$ was evaluated using 3-(4,5-dimethylthiazol-2-yl)-2,5-diphenyltetrazolium bromide (MTT)-based cell viability assays and constructing dose-response graphs as \% of untreated cells (dashed line, $100 \%$ cell viability). (C) Cells were co-treated with CQ $(20 \mathrm{ng} / \mathrm{ml})$ and various concentrations of TRAIL for $48 \mathrm{~h}$. Cell viability was measured by MTT assay and the results are presented as the calculated cell growth inhibitory ratio. Experiments were repeated three times. (D) After treatment with CQ and LY294002 for $72 \mathrm{~h}$, the expression of LC3B was shown by immunofluorescence staining. Results are means (columns) and 95\% confidence intervals (bars) of three independent experiments made in triplicate. Scale bar, $10 \mu \mathrm{m} ;{ }^{*} \mathrm{p}<0.05$.

To find additional evidence that autophagy plays a critical survival role in enabling TRAIL-refractory cell proliferation and to avoid any off-target side-effects that may confound interpretation of the results obtained with autophagy inhibitors, we tested the potent and highly sequence-specific mechanism of RNA interference (RNAi) to block LC3-dependent autophagosome formation. TRAIL-refractory cells transiently transfected with siRNA targeting Atg8/LC3 gene (the key autophagy regulatory gene). As shown in Fig. 5A,
LC3 siRNA blocked the expression of LC3B by immunoblotting and immunofluorescence staining. Moreover, we further confirmed that transfection of siRNA knock-down of LC3 was followed by exposure to $40 \mathrm{ng} / \mathrm{ml}$ TRAIL, an ineffective low-dose of TRAIL to TRAIL-refractory cells, obviously inhibited cell proliferation (Fig. 5B). Collectively, these data demonstrated clearly that hyperactivation of basal autophagy is actively involved in the development of resistance of breast cancer cells to TRAIL. 
A
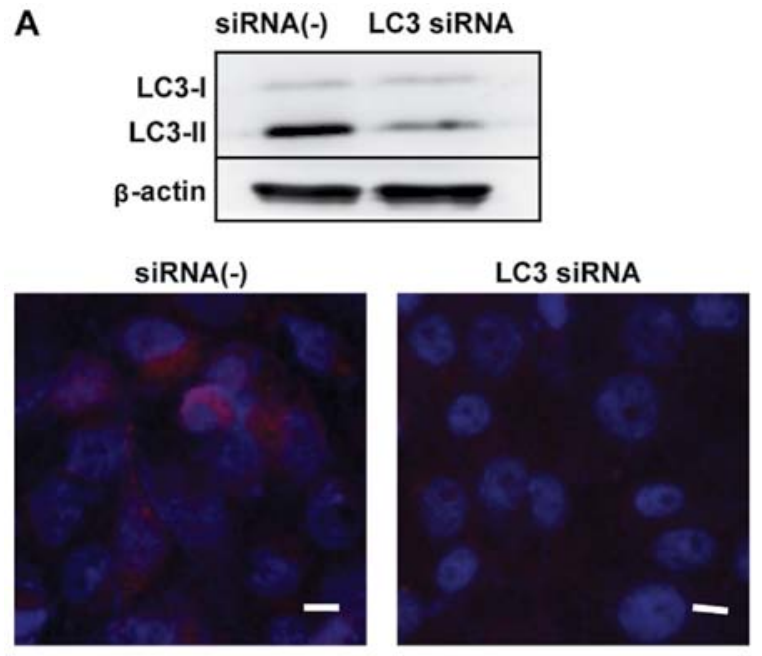

B

MDA-MB-231 TRAL-refractroy

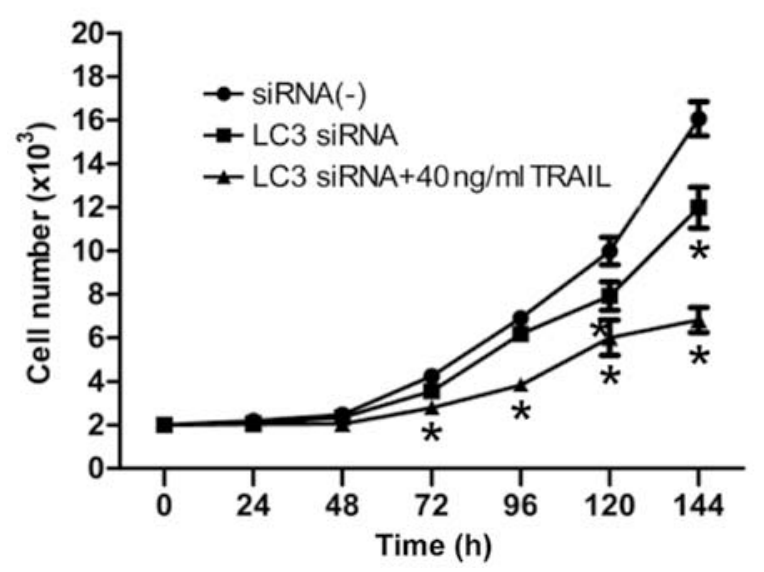

Figure 5. Changes in the cell proliferation of tumor necrosis factor-related apoptosis-inducing ligand (TRAIL)-refractory cells upon small interfering RNA (siRNA)-induced knockdown of the autophagosome marker light chain 3 (LC3). TRAIL-refractory cells were transfected with the potent and highly sequence-specific mechanism of RNA interference (RNAi) to block LC3-dependent autophagosome formation. (A) After transfection for $72 \mathrm{~h}$, non-specific negative control, and RNAi LC3-transfected cells were used for immunoblotting and immunofluorescence analyses of LC3 expression. $\beta$-actin was detected as a loading control. Scale bar, $10 \mu \mathrm{m}$. (B) After transfection for $48 \mathrm{~h}$, the proliferation rates of non-specific negative control-, RNAi LC3-transfected cells and RNAi LC3-transfected cells with $40 \mathrm{ng} / \mathrm{ml}$ TRAIL were analyzed by 3-(4,5-dimethylthiazol-2-yl)-2,5-diphenyltetrazolium bromide (MTT). Equivalent results were obtained in three independent experiments. ${ }^{*} \mathrm{P}<0.0$.

Combined TRAIL and autophagy inhibition acts synergistically to reduce TRAIL-refractory breast cancer cell viability. Thus far, we have demonstrated that TRAIL-refractory cells are exquisitely more sensitive to lysosomotropic inhibition of autophagy when compared to MDA-MB-231 parental cells. To determine whether lysosomotropic inhibition of autophagy could reverse TRAIL-refractory breast cancer cell resistance to TRAIL, TRAIL-refractory cells were treated with TRAIL (40 and $160 \mathrm{ng} / \mathrm{ml})$, CQ (20 ng/ml) for $48 \mathrm{~h}$, and their viability was assessed by MTT. The result showed that treatment with TRAIL alone led to reduction in viable cells of 3-8\%; their combination, however, yielded a more pronounced decrease in viability of $40-70 \%$ (Fig. 4B). Data analysis revealed that the decrease in cell viability was statistically significant ( $\mathrm{p} \leq 0.001$;
Fig. 4B). Therefore, CQ-mediated autophagy blockade, on its own or in combination with TRAIL significantly decreased cell viability. Co-treatment of TRAIL-refractory cells with TRAIL and autophagy blockade reduced cell viability to a greater extent than either alone.

\section{Discussion}

Since TRAIL is preferentially cytotoxic to tumor cells but not normal cells, it is considered to have strong potential as an anticancer agent. However, 50\% of tumor cell lines and the majority of primary tumors derived from cancer patients have shown resistance to TRAIL and most human breast cancer cell lines are highly resistant to TRAIL-induced apoptosis (14). Thus, the key to success of development of TRAIL receptor-targeted therapies for cancer treatment is overcoming tumor resistance.

Our laboratory has previously shown that MTDH could contribute to TRAIL resistance in breast cancer cells both in vitro and in vivo, suggesting that MTDH inhibition could be used to restore TRAIL sensitivity in TRAIL-resistant breast cancers (21). We also demonstrated that MTDH could enhance resistance to TRAIL-induced death by MTDH may overlap mechanisms: Akt activation, upregulation of Bcl-2 mediated by miR-16, downregulation of caspase- 8 , and decreased recruitment of caspase-8 into the DISC.

Autophagy is an evolutionarily conserved catabolic process. It is characterized by the appearance of autophagic vesicles and their content degraded by the cellular lysosomal system and the cell death process (22). In the cancer cells, it is still unclear if autophagy represents a survival mechanism or is involved in type II programmed cell death (PCD) (23). In addition, the common clinical anticancer drug $C Q$, inhibited autophagy and counteracted the cytotoxic effect of TRAIL.

We investigated whether the formation of autophagosomes was further enhanced in the presence of TRAIL in breast cancer cells by immunoblotting, immunofluorescence staining and MDC staining. Results from analysis indicated that the induction of autophagy was dose-dependent in MDA-MB-231 and MDA-MB-231 TRAIL-refractory cells (Fig. 2). Our results suggested that autophagy might represent a general mechanism responsible for circumventing and/or delaying TRAIL-induced cell death.

To unambiguously demonstrate that enhanced basal autophagy causally protected MDA-MB-231 TRAIL-refractory cells from cell death upon chronic exposure to TRAIL, we measured autophagosome accumulation by fluorescence microscopy of LC3B-II immunoblotting (Fig. 3). Microtubule associated protein $1 \mathrm{LC} 3$ protein, the first-known mammalian protein that is specifically associated with the autophagosomal membrane is involved in the formation of autophagosomes and its alteration from a cytosolic form LC3B-I to a lipidated form LC3B-II. Thus it has been widely used as a molecular marker of autophagosomes. The typical punctate staining that accompanied the translocation of LC3B-II from the cytosol to the autophagosome membrane was detected at high levels in MDA-MB-231 TRAIL-refractory cells, suggesting that MDA-MB-231 TRAIL-refractory cells are uniquely characterized by their ability to sustain high levels of TRAIL-induced macroautophagy without induction of 
cell death. Autophagy flux was also confirmed by fluorescence microscopy and immunoblotting of p62/SQSTM1 protein (Fig. 3A and C). p62/SQSTM1 itself is degraded by autophagy. It was shown that p62/SQSTM1 protein expression was reduced in TRAIL-refractory cells supporting the notion that the catabolic function of activated basal autophagy plays a pro-survival role in TRAIL-refractory cells. When high levels of LC3B-lipidated form associate with impairment in autophagosome maturation, this phenomenon is accompanied by a marked increase in the level of p62/SQSTM1. Conversely, increased LC3B-II levels together with a reduction of p62/SQSTM1 protein levels characterized the occurrence of autophagic flux increase (24).

MDC autophagy-specific fluorescence staining analyses and electron microscopy also confirmed this point (Fig. 3D and E). Briefly, using complementary approaches, we showed an upregulated formation of autophagosomes in the MDA-MB-231 TRAIL-refractory breast cancer cells.

Further, to determine whether autophagy induced by TRAIL could provide an indispensable role in cell survival and facilitate the development of acquired resistance to TRAIL, we pharmacologically impaired function of macroautophagosomes by using the small-molecule autophagy inhibitors LY294002 and CQ, by MTT assays (Fig. 4). The results showed that they significantly reduced cell viability in TRAIL-refractory cells. Further, combination treatment with TRAIL and CQ synergistically reduced the viability of MDA-MB-231 TRAIL-refractory cancer cells (Fig. 4), suggesting CQ was able to reverse the resistance to TRAIL and interestingly even played synergistic action with TRAIL.

LY294002 is in many cases a very effective inhibitor of class I PI3K/Akt in autophagy (25). CQ and hydroxychloroquine (HCQ) are often used in combination with chemotherapeutic drugs to enhance the efficacy of tumor cell killing. Moreover, they can suppress autophagy by inhibiting the lysosomal protease activity via neutralization of the lysosomal pH. HCQ and CQ are commonly used in suppression of autophagy (26). CQ acts on autophagosome maturation and blocks autophagic flux, as well as increases the expression of autophagic markers, including the number of autophagosomes and level of LC3-II. In fact, CQ is probably the only autophagy inhibitor currently used in clinical trials (phase I and II) in combination with various cancer therapeutic agents in different tumor types, including pancreatic, breast, colon and prostate cancer, as well as advanced solid tumors (27).

We finally used RNAi to specifically inhibit autophagy formation (Fig. 5). The knocking down of LC3 (the autophagosome membrane protein) by siRNA similarly decreased TRAIL-R cell viability as measured by MTT. In contrast to either agent alone, TRAIL and autophagy LC3 siRNA showed a profound combinatorial effect, greatly inhibited the proliferation of TRAIL-refractory cells. These combined studies not only demonstrate that development of acquired resistance to TRAIL was related to the activation of autophagy but further confirmed an active role of chemoresistance and cancer cell survival in the maintenance of TRAIL refraction. We will further explore the mechanism of autophagy in TRAIL-refractory breast cancer cells.
In summary, we have shown for the first time that autophagy plays a potent cytoprotective mechanism in the resistance of TRAIL. In addition, effectively blocking autophagosome formation could enhance TRAIL efficacy in MDA-MB-231 TRAIL-refractory cells. Clinical trials are currently ongoing which explore the combination of anti-autophagy strategies with standard therapies in human cancers (28). Our data indicate that caution is necessary in the selection of autophagy inhibitors for combination with TRAIL receptor-targeted therapies, which possibly could enhance the pro-apoptotic effect of TRAIL at doses well-tolerated by patients.

\section{Acknowledgements}

This study was supported by the National Natural Science Foundation of China (Beijing, China) (nos. 30772133, 81072150, 81172529, and 81272903) and the Shandong Science and Technology Development Plan (no. 2012GZC22115). We thank Cunzhong Yuan and Shi Yan for technical support with experiments. We also thank Yang Wang, Qiang Huo and Xia Ding for critical discussing and substantial help.

\section{References}

1. Mizushima N: Physiological functions of autophagy. Curr Top Microbiol Immunol 335: 71-84, 2009.

2. Mathew R, Karantza-Wadsworth V and White E: Role of autophagy in cancer. Nat Rev Cancer 7: 961-967, 2007.

3. Levine B and Kroemer G: Autophagy in the pathogenesis of disease. Cell 132: 27-42, 2008.

4. Eskelinen EL: The dual role of autophagy in cancer. Curr Opin Pharmacol 11: 294-300,2011.

5. Degenhardt K, Mathew R, Beaudoin B, et al: Autophagy promotes tumor cell survival and restricts necrosis, inflammation, and tumorigenesis. Cancer Cell 10: 51-64, 2006.

6. Amaravadi RK, Lippincott-Schwartz J, Yin XM, et al: Principles and current strategies for targeting autophagy for cancer treatment. Clin Cancer Res 17: 654-666, 2011.

7. Qadir MA, Kwok B, Dragowska WH, et al: Macroautophagy inhibition sensitizes tamoxifen-resistant breast cancer cells and enhances mitochondrial depolarization. Breast Cancer Res Treat 112: 389-403, 2008.

8. Wiley SR, Schooley K, Smolak PJ, et al: Identification and characterization of a new member of the TNF family that induces apoptosis. Immunity 3: 673-682, 1995.

9. Ashkenazi A, Pai RC, Fong S, et al: Safety and antitumor activity of recombinant soluble Apo2 ligand. J Clin Invest 104: 155-162, 1999.

10. Walczak H, Miller RE, Ariail K, et al: Tumoricidal activity of tumor necrosis factor-related apoptosis-inducing ligand in vivo. Nat Med 5: 157-163, 1999.

11. Mahalingam D, Szegezdi E, Keane M, de Jong S and Samali A: TRAIL receptor signalling and modulation: are we on the right TRAIL? Cancer Treat Rev 35: 280-288, 2009.

12. Abdulghani $\mathrm{J}$ and El-Deiry WS: TRAIL receptor signaling and therapeutics. Expert Opin Ther Targets 14: 1091-1108, 2010.

13. Menke C, Bin L, Thorburn J, Behbakht K, Ford HL and Thorburn A: Distinct TRAIL resistance mechanisms can be overcome by proteasome inhibition but not generally by synergizing agents. Cancer Res 71: 1883-1892, 2011.

14. Keane MM, Ettenberg SA, Nau MM, Russell EK and Lipkowitz S: Chemotherapy augments TRAIL-induced apoptosis in breast cell lines. Cancer Res 59: 734-741, 1999.

15. Yoshida T, Zhang Y, Rivera Rosado LA and Zhang B: Repeated treatment with subtoxic doses of TRAIL induces resistance to apoptosis through its death receptors in MDA-MB-231 breast cancer cells. Mol Cancer Res 7: 1835-1844, 2009.

16. Rahman M, Davis SR, Pumphrey JG, et al: TRAIL induces apoptosis in triple-negative breast cancer cells with a mesenchymal phenotype. Breast Cancer Res Treat 113: 217-230, 2009. 
17. Bossen C, Tardivel A, Willen L, et al: Mutation of the BAFF furin cleavage site impairs B-cell homeostasis and antibody responses. Eur J Immunol 41: 787-797, 2011.

18. Kim SH, Kim K, Kwagh JG, et al: Death induction by recombinant native TRAIL and its prevention by a caspase 9 inhibitor in primary human esophageal epithelial cells. J Biol Chem 279: 40044-40052, 2004.

19. Biederbick A, Kern HF and Elsässer HP: Monodansylcadaverine (MDC) is a specific in vivo marker for autophagic vacuoles. Eur J Cell Biol 66: 3-14, 1995.

20. Mariño G, Ugalde AP, Salvador-Montoliu N, et al: Premature aging in mice activates a systemic metabolic response involving autophagy induction. Hum Mol Genet 17: 2196-2211, 2008

21. Zhang N, Wang X, Huo Q, et al: The oncogene metadherin modulates the apoptotic pathway based on the tumor necrosis factor superfamily member TRAIL (Tumor Necrosis Factor-related Apoptosis-inducing Ligand) in breast cancer. J Biol Chem 288: 9396-9407, 2013

22. Klionsky DJ and Emr SD: Autophagy as a regulated pathway of cellular degradation. Science 290: 1717-1721, 2000.
23. Kelekar A: Introduction to the review series Autophagy in Higher Eukaryotes - a matter of survival or death. Autophagy 4: 555-556, 2008

24. Pankiv S, Clausen TH, Lamark T, et al: p62/SQSTM1 binds directly to Atg8/LC3 to facilitate degradation of ubiquitinated protein aggregates by autophagy. J Biol Chem 282: 24131-24145, 2007.

25. Valentim L, Laurence KM, Townsend PA, et al: Urocortin inhibits Beclin1-mediated autophagic cell death in cardiac myocytes exposed to ischaemia/reperfusion injury. J Mol Cell Cardiol 40: 846-852, 2006.

26. Mizushima N, Yoshimori T and Levine B: Methods in mammalian autophagy research. Cell 140: 313-326, 2010.

27. Sternberg CN, Donat SM, Bellmunt J, et al: Chemotherapy for bladder cancer: treatment guidelines for neoadjuvant chemotherapy, bladder preservation, adjuvant chemotherapy, and metastatic cancer. Urology 69 (Suppl 1): S62-S79, 2007.

28. Yang ZJ, Chee CE, Huang S and Sinicrope FA: The role of autophagy in cancer: therapeutic implications. Mol Cancer Ther 10: 1533-1541, 2011. 